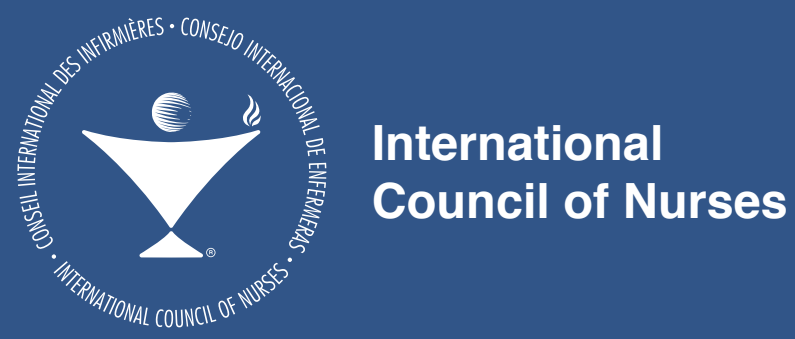

\title{
DER ICN-ETHIKKODEX
}

FÜR PFLEGEFACHPERSONEN

ÜBERARBEITET 2021 
Alle Rechte vorbehalten, auch die Übersetzung in andere Sprachen. Dieses Werk darf ganz oder teilweise unverändert und ohne vorherige schriftliche Genehmigung mit Quellenangabe nachgedruckt und weiterverbreitet werden.

Copyright @ 2021 by ICN - International Council of Nurses,

3, place Jean Marteau, 1201 Genf, Schweiz 


\section{INHALTSVERZEICHNIS}

ZWECK DES KODEX 3

PRÄAMBEL 4

DER ICN-KODEX

ANWENDUNG DER ELEMENTE DES CODES 5

ANWENDUNGSVORSCHLÄGE

für den ICN-Ethikkodex für Pflegefachpersonen 5

Pflegefachpersonen können daher: 6

VERBREITUNG

des ICN-Ethikkodex für Pflegefachpersonen 6

ELEMENTE DES KODEX

1. PFLEGEFACHPERSONEN UND PATIENTINNEN UND MENSCHEN MIT PFLEGEBEDARF

2. PFLEGEFACHPERSONEN UND DIE PRAXIS 13

3. PFLEGEFACHPERSONEN UND DER BERUF 17

4. PFLEGEFACHPERSONEN UND GLOBALE GESUNDHEIT

ICN Ethikkodex für Pflegefachpersonen

Professionelle Werte

GLOSSAR DER VERWENDETEN BEGRIFFE im ICN-Ethikkodex für Pflegefachpersonen

REFERENZEN 


und bekräftigt, zuletzt mit dieser Überprüfung und Überarbeitung 2021.

\section{ZWECK DES KODEX}

Der ICN-Ethikkodex für Pflegefachpersonen ${ }^{1}$ ist eine Erklärung der ethischen Werte, Verantwortlichkeiten und beruflichen Rechenschaftspflicht von Pflegefachpersonen und Studierenden/ Lernenden ${ }^{2}$ der Pflege. Er definiert und leitet die ethische Pflegepraxis innerhalb der verschiedenen Pflegerollen. Er ist kein Verhaltenskodex, sondern kann als Rahmen für eine ethische Pflegepraxis und Entscheidungsfindung dienen, um die von den Aufsichtsbehörden festgelegten professionellen Standards zu erfüllen.

Der ICN-Ethikkodex für Pflegefachpersonen bietet ethische Leitlinien in Bezug auf die Rollen, Pflichten, Verantwortlichkeiten, Verhaltensweisen, das professionelle Urteilsvermögen und die Beziehungen von Pflegefachpersonen zu Patientinnen ${ }^{3}$ und anderen Menschen mit Pflegebedarf, zu Kolleginnen und zu Fachpersonen anderer Berufe. Der Kodex ist grundlegend und dient zusammen mit den Gesetzen, Vorschriften und Berufsstandards der Länder, die die Pflegepraxis regeln, als Basis. Die in diesem Kodex zum Ausdruck gebrachten Werte und Verpflichtungen gelten für Pflegefachpersonen in allen Arbeitsfeldern, Rollen und Praxisgebieten.

1 Pflegefachpersonen sind Personen, die ein Pflegestudium bzw. eine Pflegeausbildung abgeschlossen haben. Sie sind berechtigt in ihrem Land den Pflegeberuf auszuüben (vgl. ICN-Statuten,

Art. 6). In der Schweiz und Deutschland sind das Pflegefachfrauen und -männer, in Österreich Gesundheits- und Krankenpfleger/innen (sowie frühere gleichwertige Berufsbezeichnungen).

2 Die Praxis der Studierenden/Lernenden in der Pflege muss in Einklang mit dem ICN-Ethikkodex stehen. Abhängig vom Ausbildungsstand, ist die Verantwortung für das Verhalten der Studierenden/ Lernenden zwischen Studierenden/Lernenden und Anleitenden geteilt. (Anm. d. Ü.: ,Studierende/Lernende' umfasst alle, die eine Ausbildung/Studium gemäß Berufeanerkennungsrichtlinie der EU oder auf vergleichbarem professionellem Niveau machen.)

3 Zur besseren Lesbarkeit wird im Text durchgehend die weibliche Form verwendet. 


\section{PRÄAMBEL}

Seit den Anfängen der organisierten Pflege Mitte des 19. Jahrhunderts und der Erkenntnis, dass die Pflege tief in den Traditionen und Praktiken von Chancengerechtigkeit und Inklusion sowie in der Wertschätzung von Vielfalt verwurzelt ist, haben Pflegefachpersonen konsequent vier grundlegende Verantwortlichkeiten anerkannt: Gesundheit fördern, Krankheiten verhüten, Gesundheit wiederherstellen sowie Leiden lindern und ein würdiges Sterben unterstützen. Der Bedarf nach Pflege ist universell.

Der Pflege inhärent ist die Achtung der Menschenrechte, einschließlich der kulturellen Rechte, des Rechts auf Leben und Wahlfreiheit, das Recht auf Würde und respektvolle Behandlung. Die Pflege ist respektvoll und uneingeschränkt in Bezug auf die Merkmale Alter, Hautfarbe, Kultur, kulturelle Zugehörigkeit, Behinderung oder Krankheit, Geschlecht, sexuelle Orientierung, Nationalität, Politik, Sprache, ethnische Zugehörigkeit, religiöse oder spirituelle Überzeugungen, rechtlicher, wirtschaftlicher oder sozialer Status.

Pflegefachpersonen werden für ihren Beitrag zur Verbesserung der Gesundheit von Einzelpersonen, Familien, Gemeinschaften und Bevölkerungsgruppen auf lokaler, nationaler und globaler Ebene geschätzt und respektiert. Sie koordinieren die Versorgung mit anderen Gesundheitsfachpersonen und weiteren involvierten Gruppen. Pflegefachpersonen zeigen professionelle ethische Werte wie Respekt, Gerechtigkeit, Empathie, Verlässlichkeit, Fürsorge, Mitgefühl, Vertrauenswürdigkeit und Integrität. 
Der ICN-Ethikkodex für Pflegefachpersonen umfasst vier Hauptelemente, die einen Rahmen für ethisches Verhalten bilden: Pflegefachpersonen und Patientinnen sowie andere Menschen mit Pflegebedarf, Pflegefachpersonen und Praxis, Pflegefachpersonen und ihre Profession sowie Pflegefachpersonen und globale Gesundheit.

\section{ANWENDUNG DER ELEMENTE DES CODES}

Die Tabellen, die auf die Beschreibung jedes Elements des Kodex folgen, sollen Pflegefachpersonen dabei unterstützen, die Standards in die Praxis umzusetzen. Zu beachten ist, dass diese Tabellen Beispiele für die wichtigsten Grundsätze der im Kodex enthaltenen Elemente aufzeigen. Sie sind weder als vollständige noch als abschließende Liste von Konzepten gedacht. Die pflegeethischen Pflichten und Werte gelten für alle Formen von pflegerischer Versorgung und in allen Rollen: Praktikerinnen, Lehrende, Studierende/Lernende, Forschende, Führungspersonen, politische Entscheidungsträgerinnen und andere. Berufsverbände werden ebenfalls durch diese Pflichten und Werte geleitet. Eine Abbildung (S. 23) verdeutlicht das Verhältnis der Werte und Pflichten für den Pflegeberuf.

\section{ANWENDUNGSVORSCHLÄGE für den ICN-Ethikkodex für Pflegefachpersonen}

Der ICN-Ethikkodex für Pflegefachpersonen ist ein Leitfaden für Maßnahmen, die auf gesellschaftlichen Werten und Bedürfnissen basieren. Er wird als Dokument nur Bedeutung haben, wenn er im Alltag der Pflege und des Gesundheitswesens in allen Arbeitsumgebungen, in denen Pflege geleistet wird, angewendet wird.

Um seinen Zweck zu erreichen, muss der Kodex von den Pflegefachpersonen in allen Aspekten ihrer Arbeit verstanden, verinnerlicht und angewendet werden. Er muss Studierenden/ Lernenden und Pflegefachpersonen während der gesamten Ausbildung und des gesamten Arbeitslebens zur Verfügung stehen. 
- Die Standards unter jedem Element des Kodex studieren.

- Persönlich überlegen, was die einzelnen Standards bedeuten. Darüber nachdenken, wie sie Ethik in der persönlichen Pflegepraxis, in der Bildung, in Forschung, Management, Führung oder Politikentwicklung anwenden können.

- Den Kodex mit Kolleginnen und anderen besprechen.

- Ein konkretes Erfahrungsbeispiel verwenden, um ethische Dilemmata und Verhaltensstandards zu identifizieren, die im Kodex beschrieben sind. Wege finden, wie der Kodex bei der Lösung von Dilemmata leitet.

- In Gruppen arbeiten, um ethische Entscheidungsfindung zu klären und einen Konsens über ethische Verhaltensstandards zu erzielen.

- Mit dem nationalen Pflegeberufsverband, Kolleginnen und anderen bei der kontinuierlichen Anwendung ethischer Standards in Pflegepraxis, Bildung, Management, Forschung und Politik zusammenarbeiten.

\section{VERBREITUNG}

\section{des ICN-Ethikkodex für Pflegefachpersonen}

Der ICN-Ethikkodex muss den Pflegefachpersonen vertraut sein, um wirksam sein zu können. Wir regen seine Verbreitung an Pflege(hoch)schulen, bei Pflegefachpersonen an ihrem Arbeitsplatz, in der Pflegefachpresse und anderen Massenmedien an. Der Kodex sollte auch bei anderen Gesundheitsberufen, der breiten Öffentlichkeit, Verbraucherinnengruppen und politischen Entscheidungsträgerinnen, Menschenrechtsorganisationen und Arbeitgeberinnen von Pflegefachpersonen verbreitet werden. Nationale Pflegeberufsverbände werden ermutigt, diesen Kodex zu übernehmen, ihn in die Landessprache(n) zu übersetzen oder inn als Grundlage für ihrer eigenen Pflegeethikkodexe zu verwenden. 

1.1 Die primäre berufliche Verantwortung der Pflegefachpersonen besteht gegenüber Menschen, die jetzt oder in Zukunft Pflege benötigen, seien es Einzelpersonen, Familien, Gemeinschaften oder Bevölkerungsgruppen (im Folgenden entweder „Patientinnen“ oder "Menschen mit Pflegebedarf“ genannt).

1.2 Pflegefachpersonen fördern ein Umfeld, in dem die Menschenrechte, Werte, Bräuche, religiösen und spirituellen Überzeugungen von Einzelnen, Familien und Gemeinschaften von allen anerkannt und respektiert werden. Die Rechte von Pflegefachpersonen sind Teil der Menschenrechte und sind zu wahren und zu schützen.

1.3 Pflegefachpersonen stellen sicher, dass Einzelne und Familien verständliche, genaue, ausreichende und rechtzeitige Informationen erhalten, angepasst an die Kultur der Patientinnen, ihre sprachlichen, kognitiven und körperlichen Bedürfnisse sowie an ihren mentalen Zustand. Sie bilden die Grundlage für die Zustimmung zur Pflege und die dazu gehörige Behandlung.

1.4 Pflegefachpersonen behandeln personenbezogene Daten vertraulich. Sie respektieren die Privatsphäre, Vertraulichkeit und die Interessen der Patientinnen bei der rechtmäßigen Erhebung, der Verwendung, dem Zugriff auf personenbezogene Daten, sowie ihrer Übermittlung, Speicherung und Offenlegung.

1.5 Pflegefachpersonen respektieren die Privatsphäre und die vertraulichen Informationen von Kolleginnen und Menschen mit Pflegebedarf, und wahren die Integrität des Pflegeberufs persönlich und in allen Medien, einschließlich der sozialen Medien.

1.6 Pflegefachpersonen teilen mit der Gesellschaft die Verantwortung, Maßnahmen zu initiieren und zu unterstützen, die den gesundheitlichen und sozialen Bedürfnissen aller Menschen gerecht werden.

4 Die beiden Begriffe ,Patientinnen' und ,Menschen mit Pflegebedarf' werden austauschbar verwendet. Die beiden Begriffe beziehen sich auf Patientinnen, Familien, Gemeinschaften und Bevölkerungsgruppen mit Pflegebedarf. Die Arbeitsfelder beinhalten Krankenhäuser, ambulante und Gemeindepflege, Primärversorgung, Öffentliche Gesundheit, Gesundheit von Bevölkerungsgruppen, Langzeitpflege, Pflege im Justizvollzug, akademische Institutionen und Behörden und sind nicht auf Settings begrenzt. 
1.7 Pflegefachpersonen setzen sich für Chancengerechtigkeit und soziale Gerechtigkeit bei der Ressourcenzuteilung, dem Zugang zu Gesundheitsversorgung und anderen sozialen und wirtschaftlichen Dienstleistungen ein.

1.8 Pflegefachpersonen zeigen professionelle Werte wie Respekt, Gerechtigkeit, Verlässlichkeit, Fürsorge, Mitgefühl, Empathie, Vertrauenswürdigkeit und Integrität. Sie unterstützen und respektieren die Würde und die universellen Rechte aller Menschen, einschließlich Patientinnen, Kolleginnen und Familien.

1.9 Pflegefachpersonen fördern eine Sicherheitskultur im Gesundheitswesen, indem sie Risiken für die Menschen oder die sichere Pflegepraxis in jeder Pflegeumgebung erkennen und aktiv angehen. Sie gewährleisten eine sichere Versorgung in der Praxis, bei Dienstleistungen und in Arbeitsumfeldern.

1.10 Pflegefachpersonen bieten eine "evidence-informed" und personenzentrierte Pflege. Sie anerkennen und verwenden die Werte und Prinzipien der primären Gesundheitsversorgung und -förderung über die gesamte Lebensspanne hinweg.

1.11 Pflegefachpersonen stellen sicher, dass der Einsatz von Technologie und wissenschaftlichen Fortschritten mit der Sicherheit und den Rechten von Menschen vereinbar sind. Bei künstlicher Intelligenz oder Geräten wie Robotern oder Drohnen sorgen Pflegefachpersonen dafür, dass die Pflege personenzentriert bleibt und solche Geräte menschliche Beziehungen unterstützen und nicht ersetzen.

\section{Anwendung der Elemente des Kodex \#1: PFLEGEFACHPERSONEN} UND PATIENTINNEN ODER MENSCHEN mit Pflegebedarf

\section{Pilegefachpersonen \\ und FUhrungspersonen in der Pilege}

Bieten Menschen eine gezielte, kulturell angemessene Pflege, die die Menschenrechte respektiert und sensibel ist für die Werte, Bräuche und Überzeugungen der Menschen, ohne Vorurteile oder ungerechte Diskriminierung.
Lehrende

und Forschende

Nehmen in die Lehrpläne Inhalte zu kulturellen Normen, Sicherheit und Kompetenz, Ethik, Menschenrechten, Gleichbehandlung, Menschenwürde, Gerechtigkeit, Ungleichheiten und Solidarität als Grundlage für den Zugang zur Gesundheitsversorgung auf. Entwerfen Studien zur Erforschung von Menschenrechtsfragen.
Nationale Pflegeberufisverbände

Entwickeln Positionspapiere, Praxisstandards und Leitlinien, die Menschenrechte und ethische Standards unterstützen. 
Piflegefachpersonen

und Führungspersonen in der Pillege

Nehmen an Fortbildungen zu ethischen Fragen, ethischem Denken und ethischem Verhalten teil.

Fördern einen offenen Dialog zwischen allen Beteiligten.

Stellen die ausreichende Aufklärung für die Einwilligung zu Pflege und/oder medizinischer Versorgung sicher. Das schließt das Recht ein, Behandlungen zu wählen oder abzulehnen.

Setzen professionelles ethisches Urteilsvermögen bei der Verwendung von elektronischen oder auf Papier geschriebenen Informationen, Gesundheitsakten und Meldesystemen ein, um den Schutz der Menschenrechte, der Vertraulichkeit und der Privatsphäre in Übereinstimmung mit den Wünschen der Patientinnen und der Sicherheit der Gemeinschaft, in Übereinstimmung mit den relevanten Gesetzen sicher zu stellen

\section{Lehrende und Forschende}

Gestalten Lehrpläne, die aktuelle, von Experten begutachtete und veröffentlichte Vorgehensweisen zur Pflegeethik enthalten. Bieten Lehr- und Lernmöglichkeiten für ethische Fragen, ethische Prinzipien und Argumentationen sowie für ethische Entscheidungsfindung an. Dazu gehört die Achtung von Autonomie, NichtSchaden (nonmaleficence), Gutes tun (beneficence) und Gerechtigkeit (Fairness).

Informieren über den Respekt der Autonomie, Zustimmung nach Aufklärung, Privatsphäre und Vertraulichkeit. Respektieren das Recht von Studienteilnehmenden, die Teilnahme an Studien ohne Nachteile zu verweigern oder abzubrechen.

\section{Erarbeiten Lehrpläne, die} Genauigkeit, Vertraulichkeit und Datenschutz bei der Verwendung von Medien, Berichterstattungs- und Aufzeichnungssystemen für Bild, Ton und Kommentare beinhalten. Machen sich mit der Verwendung der erforderlichen Dokumentation für extreme Notfälle vertraut.
Veröffentlichen Leitlinien für die Einwilligung von Studienteilnehmenden in Bezug auf Einwilligung nach Aufklärung, in öffentlichen Stellungnahmen, wichtigen Dokumentationen und in kontinuierlicher Fort- und Weiterbildung.

\section{Erarbeiten Leitlinien und} Praxisstandards für die angemessene Nutzung von Informations- und Meldesystemen, die den Schutz der Menschenrechte, die Vertraulichkeit und die Privatsphäre garantieren, Erarbeiten Leitlinien und Praxisstandards für die Nutzung von vorgeschriebenen Meldemechanismen bei Gefährdungen der öffentlichen Gesundheit und extremen Notfällen. 


\section{Pflegefachpersonen \\ und Fuhrungspersonen in der Pflege}

\section{Informieren die}

zuständigen Vorgesetzten und/oder Behörden über

Risiken, unangemessenes Verhalten oder Missbrauch von Technologien, die die Sicherheit von Personen gefährden, und liefern Fakten, die dies belegen.

\section{Pflegefachpersonen} müssen in die Entwicklung von Technologien einbezogen werden und Risiken im Zusammenhang mit technologischen und wissenschaftlichen Fortschritten beobachten und melden.

\section{Erfüllen die ethischen Verpflichtungen und Verantwortlichkeiten von Pflegefachpersonen und bekräftigen aktiv die Werte und Ideale des Berufs.}

Entwickeln und

überwachen die Sicherheit am Arbeitsplatz.

\section{Lehrende und Forschende}

Nehmen Maßnahmen, die eine sichere Pflege fördern, Würde und Rechte respektieren und neue Technologien berücksichtigen, in die Lehrpläne auf und forschen dazu.

Nehmen berufliche Werte und Ideale, ethische Verantwortlichkeiten und Verpflichtungen sowie ethische Bezugssysteme und Weltanschauungen in die Lehrpläne auf.

Tragen zu ethischen Forschungsrichtlinien bei und verbreiten diese mit Nachdruck.

Entwerfen Studien

zur Erforschung von

Menschenrechtsthemen.

\section{Lehren und fördern das}

Erkennen von Kennzeichen und Risikofaktoren, und unterrichten Fähigkeiten, um ein gesundes, sicheres und nachhaltiges Praxisumfeld für alle im Gesundheitswesen zu gewährleisten.

\section{Nationale
Pflegeberufisverbände}

Setzen sich bei

Regierungen,

Gesundheitsorganisationen,

Medizinprodukte- und

Pharmaunternehmen

dafür ein, dass

\section{Pflegefachpersonen}

während der Forschung

und Entwicklung von

Technologien für

Patientinnen einbezogen sind.

\section{Bringen die Werte und} Ideale der Pflege in ihren Grundlagendokumenten zum Ausdruck und integrieren sie in die nationalen Ethikkodexe für Pflegefachpersonen.

Nehmen Einfluss auf Arbeitgeberinnen, um gesunde und sichere Arbeitsplätze für Pflegefachpersonen und andere Mitarbeitende des Gesundheitswesens zu fördern. Erstellen Richtlinien, die eine sichere Umgebung und gesunde Gemeinschaften gewährleisten.

Setzen sich für klare, zugängliche, transparente und wirksame

Meldeverfahren zum

Schutz von Gesundheit und

Sicherheit ein. 


\section{PFLEGEFACHPERSONEN UND DIE PRAXIS}

2.1 Pflegefachpersonen sind persönlich verantwortlich und rechenschaftspflichtig für die ethische Pflegepraxis und die Aufrechterhaltung ihrer Kompetenzen, durch kontinuierliche berufliche Weiterentwicklung und lebenslanges Lernen

2.2 Pflegefachpersonen halten ihre fachlichen Kompetenzen aktuell, damit sie ihre Fähigkeit, eine qualitativ hochwertige und sichere Pflege zu gewährleisten, nicht gefährden.

2.3 Pflegefachpersonen praktizieren innerhalb der Grenzen ihrer individuellen Kompetenz und dem gesetzlich vorgegebenen Verantwortungsbereich. Sie verwenden professionelles Urteilsvermögen, wenn sie Verantwortung übernehmen und delegieren.

2.4 Pflegefachpersonen wertschätzen ihre eigene Würde, ihr Wohlbefinden und ihre Gesundheit. Um dies zu erreichen, braucht es positive Arbeitsumgebungen, die geprägt sind von beruflicher Anerkennung, Bildung, Reflexion, Unterstützungsstrukturen, angemessener Ressourcenausstattung, solide Managementpraktiken sowie Gesundheitsschutz und Arbeitssicherheit.

2.5 Pflegefachpersonen legen jederzeit ein angemessenes Verhalten an den Tag. Sie repräsentieren den Beruf positiv und stärken sein Image und das öffentliche Vertrauen. In ihrer beruflichen Rolle erkennen und berücksichtigen Pflegefachpersonen Grenzen der persönlichen Beziehungen.

2.6 Pflegefachpersonen teilen ihr Wissen und ihre Expertise und geben Feedback, um Studierende/Lernende, Berufsanfängerinnen, Kolleginnen und Angehörige anderer Gesundheitsberufe anzuleiten und zu unterstützen.

2.7 Pflegefachpersonen sind Fürsprecherinnen für Betroffene und pflegen eine Praxiskultur, die ein ethisches Verhalten und den offenen Dialog fördert.

2.8 Pflegefachpersonen haben die Möglichkeit, die Teilnahme an bestimmten Prozeduren oder pflege- oder gesundheitsbezogener Forschung aus Gewissensgründen abzulehnen. Sie müssen respektvoll und rechtzeitig handeln, um sicherzustellen, dass die Menschen eine ihren individuellen Bedürfnissen angemessene Pflege erhalten. 
2.9 Pflegefachpersonen schützen das Recht einer Person, den Zugriff auf ihre persönlichen, gesundheitsbezogenen und genetischen Informationen zu erteilen und zu widerrufen. Sie schützen die Nutzung, Privatsphäre und Vertraulichkeit beim Umgang mit genetischen Informationen und humanen Genomtechnologien.

2.10 Pflegefachpersonen ergreifen geeignete Maßnahmen, um Einzelpersonen, Familien, Gemeinschaften und Bevölkerungsgruppen zu schützen, wenn deren Gesundheit durch eine Kollegin, eine andere Person, Regelwerke, oder die Anwendung oder den Missbrauch von Technologie gefährdet wird.

2.11 Pflegefachpersonen beteiligen sich aktiv an der Förderung der Sicherheit der Patientinnen. Sie fördern ethisches Verhalten, wenn Fehler oder Beinahe-Fehler auftreten. Sie melden sich zu Wort, wenn die Sicherheit der Patientinnen gefährdet ist. Sie setzen sich für Transparenz ein und arbeiten mit anderen zusammen, um das Fehlerpotenzial zu reduzieren.

2.12 Pflegefachpersonen sind für die Korrektheit und Vollständigkeit von Daten verantwortlich, um ethische Standards der Pflege zu unterstützen und zu fördern.

Anwendung der Elemente des Kodex \#2: PFLEGEFACHPERSONEN UND DIE PRAXIS

\section{Pflegefachpersonen und FÜhrungspersonen in der Pilege}

Entwickeln sich durch Lesen und Lernen beruflich weiter. Fordern Fort- und Weiterbildungen ein und nehmen daran teil, um Kenntnisse und Fähigkeiten zu erweitern.

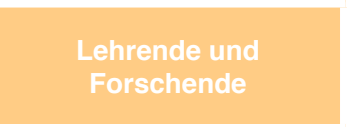

Lehren und fördern den Wert und die Verpflichtung zu lebenslangem Lernen und die Kompetenz zu praktizieren. Erkunden aktuelle Konzepte und innovative Lehrmethoden für Theorie und Praxis.
Nationale Pilegeberufisverbände

Entwickeln mithilfe von Zeitschriften, Medien, Konferenzen und Fernunterricht eine Reihe von kontinuierlichen Bildungsmöglichkeiten, die die Fortschritte in Theorie und Pflegepraxis spiegeln. 
Pilegefachpersonen

und Fuhrungspersonen in der Pflege

Engagieren sich kontinuierlich für Fortund Weiterbildungen und beteiligen sich an der Gestaltung des Arbeitsplatzes, den Systemen zur Leistungsbeurteilung, der Bewertung und systematischen Erneuerung der Berufszulassung. Sie überwachen, fördern und bewerten die Aufrechterhaltung der pflegerischen Kompetenzen des Pflegepersonals.

Streben eine gute Balance zwischen Leben und Arbeit, die kontinuierliche persönliche Entwicklung und einen gesunden Lebensstil an.

Fördern die interprofessionelle Zusammenarbeit zur Bewältigung von Konflikten und Spannungen. Fördern ein Umfeld gemeinsamer ethischer Werte.

Um die

Versorgungsqualität und -sicherheit zu verbessern, muss die Angst vor Repressalien beseitigt werden. Dadurch wird eine offenere, transparentere Kultur schaffen, die wichtige Gespräche zur Förderung der Gesundheit für alle umfasst.

\section{Lehrende und Forschende}

Führen Forschung zum Zusammenhang zwischen kontinuierlichem Lernen und Aufrechterhaltung der pflegerischen Kompetenzen durch und verbreiten die Resultate.
Lehren Verpflichtungen sich selbst und Patientinnen gegenüber, die Bedeutung der Aufrechterhaltung der pflegerischen Kompetenzen und die Anwendung von „evidenceinformed" Pflege. Schließen die Förderung von Resilienz am Arbeitsplatz in die Lehrpläne ein.

Vermitteln Methoden und Fähigkeiten zur Situationsbeurteilung und des Konfliktmanagements und lehren die Rollen und Werte anderer Gesundheitsfachgebiete.
Setzen sich für Arbeitsumgebungen ein, die Standards für einen gesunden Lebensstil für Pflegefachpersonen fördern.

Erstellen Leitlinien zu sicheren und angemessenen Arbeitsbedingungen für Pflegefachpersonen.

Informieren andere Berufsgruppen und die Öffentlichkeit über die Rolle der Pflegefachpersonen und die Werte des Pflegeberufs. Fördern ein positives Image der Pflege. Setzen sich für Arbeitsumgebungen und -bedingungen ein, die frei sind von Missbrauch, Belästigung und Gewalt. 


\section{Pillegefachpersonen und Fuhrungspersonen in der Pflege}

Entwickeln angemessene berufliche Beziehungen

zu Patientinnen und Kolleginnen; urteilen professionell, lehnen Geschenke oder Bestechungsgüter ab und vermeiden Interessenkonflikte.

\section{Gewährleisten die} Kontinuität bei der Pflege einer Patientin, wenn sie eine Handlung aufgrund von möglichem Schaden oder aus persönlichen

\section{Gewissensgründen} verweigern

\section{Lehrende und Forschende}

Bewahren und lehren berufliche Grenzen und Fähigkeiten, um sie zu gewährleisten. Lehren die Identifizierung von Methoden zur Vermeidung von Interessenkonflikten.

Ermutigen zur

Selbstreflexion und lehren Rahmenbedingungen und Prozesse zur Verweigerung aus Gewissensgründen.

\section{Nationale} Pfilegeberufisverbände

Setzen Standards für berufliche Grenzen und etablieren Prozesse zur Äußerung von Anerkennung und Dankbarkeit.

Entwickeln Standards
und Richtlinien für
die Verweigerung der
Teilnahme an bestimmten
medizinischen Verfahren.
Nehmen Leitlinien zur
Verweigerung aus
Gewissensgründen in die
nationalen Ethikkodexe auf.


3.1 Pflegefachpersonen übernehmen die führende Rolle bei der Festlegung und Umsetzung „evidence-informed“, akzeptabler Standards der klinischen Pflegepraxis, des Managements, der Forschung und der Ausbildung.

3.2 Pflegefachpersonen und Pflegewissenschaftlerinnen sind aktiv in der Erweiterung des forschungsbasierten, aktuellen Berufswissens, das eine „evidence-informed“" Praxis unterstützt.

3.3 Pflegefachpersonen sind aktiv bei der Entwicklung und Erhaltung eines Kerns von beruflichen Werten.

3.4 Pflegefachpersonen beteiligen sich durch ihre Berufsorganisationen an der Schaffung einer positiven und konstruktiven Arbeitsumgebung, welche die klinische Pflege, die Ausbildung, die Forschung, das Management und die Führung umfasst. Dazu gehören Umgebungen, die es Pflegefachpersonen ermöglichen, ihren Verantwortungsbereich optimal auszufüllen und eine sichere, effektive und rechtzeitige Gesundheitsversorgung zu gewährleisten. Dies unter Arbeitsbedingungen, die für Pflegefachpersonen sicher, sowie sozial und wirtschaftlich gerecht sind.

3.5 Pflegefachpersonen tragen zu einer positiven und ethischen Arbeitsumgebung bei und setzen sich gegen unethische Praktiken und Einstellungen ein. Pflegefachpersonen arbeiten mit Pflegekolleginnen, anderen (Gesundheits-)Disziplinen und relevanten Gruppen zusammen. Sie beteiligen sich an der Erstellung, Durchführung und Verbreitung von „peer-reviewed“ und ethisch verantwortungsvoller Forschung und Praxisentwicklung im Kontext von Patientinnenversorgung, Pflege und Gesundheit.

3.6 Pflegefachpersonen beteiligen sich an der Erstellung, Verbreitung und Anwendung von Forschung, die die Ergebnisse für Einzelpersonen, Familien und Gemeinschaften verbessern.

3.7 Pflegefachpersonen bereiten sich auf Notfälle, Katastrophen, Konflikte, Epidemien, Pandemien, soziale Krisen und Situationen mit knappen Ressourcen vor und reagieren darauf. Die Sicherheit der Menschen, die Pflege erhalten, liegt in der Verantwortung der einzelnen Pflegefachpersonen und der Führungspersonen von Gesundheitssystemen und -organisationen. Das beinhaltet die Bewertung von Risiken und die Entwicklung, Umsetzung und Planung von Ressourcen, um diese zu minimieren. 
Pilegefachpersonen

und Fuhrungspersonen in der Pflege

Arbeiten mit Kolleginnen

zusammen, um

die Durchführung,

Verbreitung und Nutzung

von Forschung zu

Patientinnenversorgung,

Pflege und Gesundheit zu unterstützen.

Fördern die Mitwirkung

in nationalen

Pflegeberufsverbänden,

um Solidarität und

Zusammenarbeit zu

schaffen, und um günstige

sozioökonomische und

Arbeitsbedingungen für

Pflegefachpersonen zu

fördern.

\section{Üben ethische \\ Verhaltensweisen ein und entwickeln Strategien, um in aufkommenden Krisen wie Pandemien oder Konflikten mit moralischem Stress umzugehen.}

Entwickeln Richtlinien für

Probleme am Arbeitsplatz wie Mobbing, Gewalt, sexuelle Belästigung, Erschöpfung, Sicherheit und Management von lokalen Zwischenfällen. Nehmen an Studien zu Ethik und ethischen Fragen am Arbeitsplatz in jedem Umfeld teil.

\section{Lehrende und} Forschende

\section{Lehren}

Forschungsmethodik, Ethik und Evaluation. Führen, verbreiten, nutzen und werten Forschung aus, um Pflegewissen zu studieren und zu erweitern.

Betonen bei den Studierenden/Lernenden das Wesen, die Funktion und die Bedeutung von Pflegeberufsverbänden und der internationalen Pflegezusammenarbeit.

Bereiten die Studierenden/ Lernenden mit einer umfassenderen Vision von Solidarität und Gemeinwohl auf eine lokale Reaktion auf globale Probleme vor. Berücksichtigen dabei gesundheitliche Ungleichheiten, insbesondere von Kindern, gebrechlichen älteren Menschen, Gefangenen, wirtschaftlich Benachteiligten, Opfern von Menschenhandel, Vertriebenen und Flüchtlingen.

Lehren das Erkennen
von ungesunden
Arbeitsumgebungen
und Fähigkeiten, um
widerstandsfähige
und gesunde
Arbeitsplatzgemeinschaften
zu entwickeln. Führen
berufsübergreifend
Forschung zu ethischen
Fragen am Arbeitsplatz
durch.

\section{Nationale \\ Piflegeberufsverbände}

\section{Entwickeln}

Positionspapiere, Leitlinien, Strategien und Standards, die auf der Grundlage von Pflegeforschung und wissenschaftlichen Untersuchungen basieren.

Vermitteln die Bedeutung der Mitgliedschaft in Pflegeberufsorganisationen und fördern die Mitwirkung in nationalen Pflegeberufsverbänden.

Arbeiten mit globalen Organisationen zusammen, um aktuelle und aufkommende Probleme sozialer Gerechtigkeit anzugehen.

Nehmen Einfluss, üben Druck aus und verhandeln für faire und angemessene Arbeitsbedingungen.

Entwickeln

Positionspapiere und Richtlinien, um Probleme am Arbeitsplatz anzugehen. 
Pilegefachpersonen und Fuhrungspersonen in der Pilege

Bereiten sich auf Notfälle, Katastrophen, Konflikte, Epidemien, Pandemien und Situationen knapper Ressourcen vor und reagieren darauf.

Diskriminieren Kolleginnen aus anderen Kulturen und Ländern nicht, weder aufgrund ihrer Nationalität, noch wegen ihrer ethnischen oder kulturellen Zugehörigkeit oder Sprache.

\section{Lehrende und Forschende}

Stellen sicher, dass die Lehrpläne wesentliche Elemente der Betreuung von Menschen und Bevölkerungsgruppen in risikoreichen und herausfordernden Umgebungen enthalten.

Vermitteln die Grundsätze des Verhaltenskodex der WHO zur internationalen Anwerbung von Gesundheitspersonal, um die ethische Anwerbung von Pflegefachpersonen zu unterstützen.

\section{Nationale Pflegeberufisverbände}

Fordern und setzen sich bei Regierungen und Gesundheitsorganisationen dafür ein, dass die Gesundheit, die Sicherheit und das Wohlbefinden des Gesundheitspersonals während der Bewältigung von gesundheitlichen Notfällen priorisiert und geschützt wird.

Fördern die ethische Anwerbung von Pflegefachpersonen und arbeiten mit Regierungsund Zulassungsbehörden zusammen, um Beschäftigungshindernisse für Pflegefachpersonen aus dem Ausland abzubauen. 
4.1 Pflegefachpersonen erachten die Gesundheitsversorgung als Menschenrecht und bekräftigen das Recht auf universellen Zugang zur Gesundheitsversorgung für alle.

4.2 Pflegefachpersonen wahren die Würde, die Freiheit und den Wert aller Menschen und wenden sich gegen alle Formen der Ausbeutung, wie Menschenhandel und Kinderarbeit.

4.3 Pflegefachpersonen übernehmen eine Führungsrolle oder tragen zu einer soliden gesundheitspolitischen Entwicklung bei.

4.4 Pflegefachpersonen tragen zur Gesundheit der Bevölkerung bei und arbeiten auf die Erreichung der Nachhaltigen Entwicklungsziele der Vereinten Nationen (SDG) hin.

4.5 Pflegefachpersonen anerkennen die Bedeutung der sozialen Determinanten von Gesundheit. Sie tragen zu entsprechenden Richtlinien und Programmen bei und setzen sich dafür ein.

4.6 Pflegefachpersonen setzen sich gemeinsam dafür ein, die natürliche Umwelt zu erhalten, zu stärken und zu schützen. Sie sind sich der gesundheitlichen Folgen der Umweltzerstörung, z.B. aufgrund des Klimawandels, bewusst. Sie treten für Initiativen ein, die umweltschädliche Praktiken reduzieren, um Gesundheit und Wohlbefinden zu fördern.

4.7 Pflegefachpersonen arbeiten mit anderen Gesundheits- und Sozialberufen und der Bevölkerung zusammen, um die Grundsätze der Gerechtigkeit zu wahren, indem sie die Verantwortung für Menschenrechte, Chancengerechtigkeit und Fairness fördern und sich für das öffentliche Wohl und für einen gesunden Planeten einsetzen.

4.8 Pflegefachpersonen arbeiten länderübergreifend zusammen, um die globale Gesundheit zu entwickeln und zu erhalten, und Richtlinien und Grundsätze dafür sicherzustellen. 
Pilegefachpersonen

und Führungspersonen in der Pflege

Beteiligen sich an

Bemühungen zur

Einhaltung der

Menschenrechte,

wie der Prävention

und Aufdeckung von

Menschenhandel,

helfen vulnerablen

Bevölkerungsgruppen,

bieten allgemeine Bildung

an und lindern Hunger und

Armut.

\section{Informieren sich und ihre Kolleginnen über globale Gesundheit, einschließlich aktueller und aufkommender Technologien. \\ Setzen sich für den ethischen Einsatz von Technologie und wissenschaftlichen Fortschritten ein, damit sie mit Sicherheit, Würde, Privatsphäre, \\ Vertraulichkeit und Menschenrechten vereinbar sind.}

Erwerben und verbreiten Wissen über die negativen Auswirkungen des Klimawandels auf die Gesundheit der Menschen und den Planeten.

\section{Lehrende und Forschende}

Stellen sicher, dass die Lehrpläne Menschenrechte

\section{Nachhaltige}

Entwicklungsziele (SDG), universellen Zugang zur Gesundheitsversorgung, zu kulturell angemessener Pflege, zu ziviler Verantwortung, Gleichbehandlung sowie sozialer und ökologischer Gerechtigkeit enthalten.

\section{Suchen nach}

Möglichkeiten, um die kurzund langfristigen ethischen Konsequenzen des Einsatzes verschiedener Technologien und neu entstehender Praktiken zu bewerten, einschließlich innovativer Geräte, Robotik, Genetik und Genomik, Stammzelltechnologien und Organspenden.

Lehren über die Fakten und Folgen des Klimawandels auf die Gesundheit und die vielen Möglichkeiten, Klimagesundheit auf politischer und institutioneller Ebene zu unterstützen.

\section{Nationale} Pilegeberuifverbände

Arbeiten mit den Behörden zusammen, die die Pflege regeln, ebenso mit Freiwilligenorganisationen und globalen Akteuren, um Positionspapiere und Richtlinien zu entwickeln, die Menschenrechte, Umweltgerechtigkeit und internationalen Frieden unterstützen.

Tragen zu Gesetzen und politischen Strategien zur ethischen Nutzung von Technologie und wissenschaftlichen Fortschritten bei, die an die gesundheitlichen und sozialen Normen und den Kontext des Landes angepasst sind.

Beteiligen sich an der Entwicklung von Rechtsvorschriften, um die Umweltauswirkungen von Krankenhäusern und der Gesundheitsbranche zu verringern und sprechen den Klimawandel an, der sich negativ auf die Gesundheit der Bevölkerung auswirkt. 


\section{Pflegefachpersonen \\ und Führungspersonen in der Pilege}

Unterstützen die ethische und kompetente Nutzung von sozialen Medien und Technologien, um die Gesundheit der Bevölkerung, im Einklang mit den Werten des Pflegeberufs, zu verbessern.
Handeln bei lokalen und globalen Themen, die die Gesundheit betreffen, wie Armut, Nahrungsmittelsicherheit, Wohnsituation, Migration, Geschlecht, Klasse, kulturelle und ethnische Zugehörigkeit, Umweltgesundheit, menschenwürdige Arbeit und Bildung.

Binden die Konzepte von Frieden, Friedensdiplomatie und Friedensförderung in die tägliche Praxis ein.

\section{Lehrende und Forschende}

Beteiligen sich an der Entwicklung, Implementierung und Bewertung neuer und aufkommender Technologien, einschließlich der sozialen Medien, für Präventionsinitiativen, die öffentliche Gesundheitserziehung, sowie die Gesundheit und das Wohlergehen der Bevölkerung. Bereiten Lehrpläne vor und engagieren sich in der Forschung zur Unterstützung der Nachhaltigen Entwicklungsziele (SDG) der Vereinten Nationen.

Informieren über gesellschaftspolitische und wirtschaftliche Themen, die sich auf die Gesundheit auswirken, einschließlich Geschlecht, kultureller und ethnischer Zugehörigkeit, Kultur, Ungleichheit und Diskriminierung. Erforschen gesellschaftspolitische Faktoren, die zur Gesundheit und Krankheit des Einzelnen und der Bevölkerung beitragen.

Lehren und forschen zu Friedensdiplomatie und Friedensförderung in Gemeinschaften und weltweit.

\section{Nationale} Pilegeberufisverbände

Aktualisieren den Wissensstand und erhöhen das Bewusstsein für die Nachhaltigen Entwicklungsziele (SDG) der Vereinten Nationen für die Gesundheit der Bevölkerung und entwickeln aktiv Strategien für die Mitwirkung der Pflege zur Erreichung dieser Ziele

Arbeiten mit anderen nationalen und internationalen Pflegeberufsorganisationen zusammen, um Richtlinien und Gesetze zu formulieren, die sich mit den sozioökonomischen Determinanten von Gesundheit befassen.

Arbeiten global, national und regional mit Regierungen und Behörden zusammen, um die Ziele des globalen Friedens und der Gerechtigkeit voranzutreiben und die Ursachen von Krankheiten zu lindern. 


\section{ICN Ethikkodex für Pflegefachpersonen}

\section{Professionelle Werte}

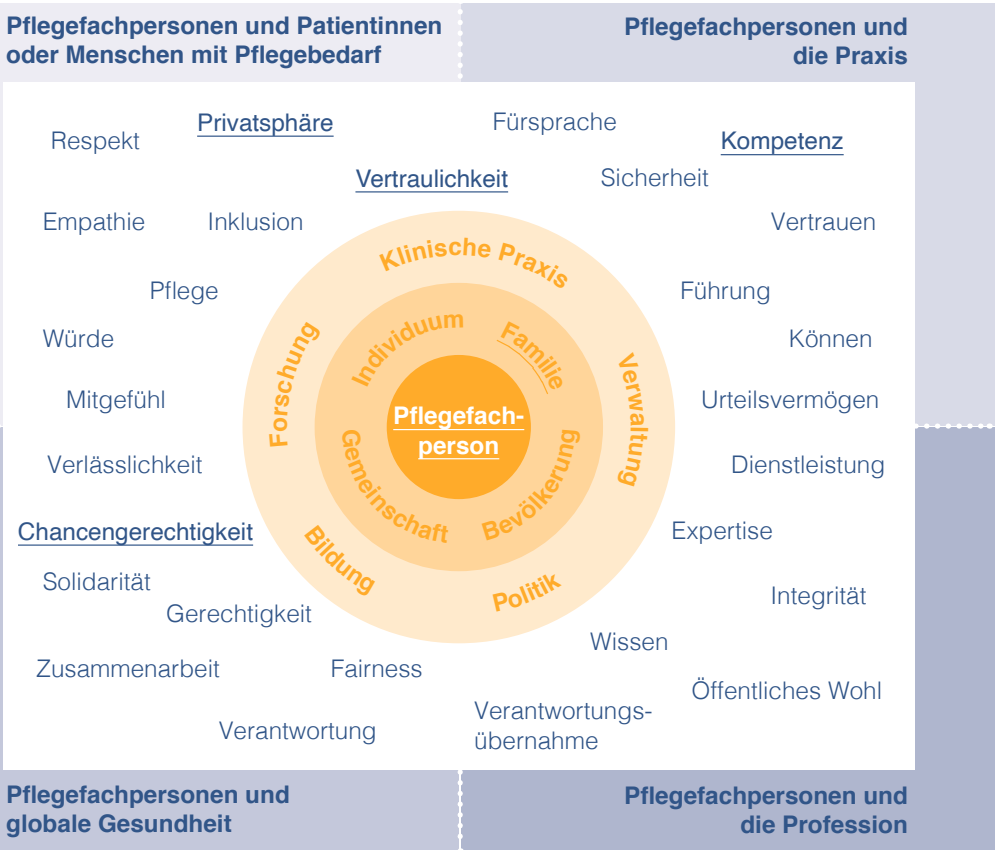





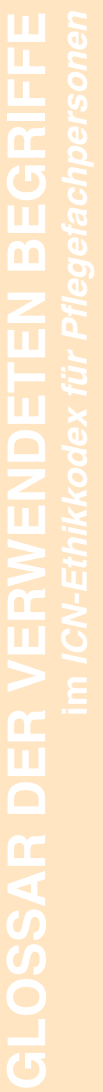



Aufrechterhaltung der pflegerischen Kompetenzen

Ethik

Evidence-informed Praxis

Familie

Fürsprecherin

Führungspersonen in der Pflege

Genetik

Genomik

Gleichbehandlung

Kolleginnen

Kompetenz
Die Fähigkeiten, das Wissen, die Gesundheit und den Charakter haben, um die eigene Arbeit sicher und effektiv auszuführen. (UK Nursing and Midwifery Council 2021)

Ein Teilgebiet der Philosophie. Angewandte normative Ethik wird im Gesundheitswesen und in der Berufsethik am häufigsten verwendet. Sie hilft, das „Sollte" auf sozialer, gemeinschaftlicher oder individueller Ebene zu bestimmen. Sie befasst sich auch mit breiten sozialen Themen wie Menschenrechten, globaler Zusammenarbeit, Klimawandel, globalen Pandemien, soziostrukturellen Ungleichheiten.

„Evidence-informed" Praxis ist ein Prozess, um fundierte klinische Entscheidungen zu treffen. Forschungsevidenz wird mit klinischer Erfahrung, Werten von Patientinnen, Präferenzen und den bestehenden Verhältnissen zusammengeführt. (Woodbury \& Kuhnke 2014)

Eine soziale Einheit, die aus Mitgliedern besteht, die durch Blut, Verwandtschaft, emotionale oder rechtliche Beziehungen verbunden sind.

Unterstützt aktiv eine richtige und gute Sache; unterstützt Andere darin, für sich selbst zu sprechen oder im Namen anderer zu sprechen, die nicht für sich selbst sprechen können. Die Interessenvertretung wird immer mit Zustimmung der Person selbst durchgeführt.

Eine Führungsperson in der Pflege ist für den täglichen Betrieb einer Pflegeeinheit und die Beaufsichtigung des Pflegepersonals in einer bestimmten Einheit oder Abteilung verantwortlich.

Die Untersuchung einzelner Gene, genetischer Varianten und Vererbung in Organismen.

Die Untersuchung des vollständigen Gen-Satzes einer Person, des Genoms, um Varianten zu finden, die die Gesundheit, die Reaktion auf Arzneimittel, die Interaktionen zwischen den Genen oder mit der Umwelt beeinflussen.

Gleichbehandlung ist ein Aspekt der sozialen Gerechtigkeit. Es bezieht sich auf das Fehlen von systemischen Nachteilen, die zu gesundheitlichen Ungleichheiten für bestimmte Bevölkerungsgruppen führen.

Gleichbehandlung ist für die volle Anerkennung der Menschenrechte von wesentlicher Bedeutung.

Pflegefachpersonen und andere Gesundheits- und nicht gesundheitsbezogene Arbeitnehmerinnen und Fachleute.

Das integrierte Wissen, die Fähigkeiten, das Urteilsvermögen und die Eigenschaften, die von einer Pflegefachperson verlangt werden, um in einer bestimmten Rolle und Umgebung sicher und ethisch zu praktizieren. 
Menschenrechte

Nachhaltige

Entwicklungsziele (SDG)

Nationale
Pflegeberufsverbände

Personenzentrierte

Pflege

Persönliche Angaben

Pflegefachperson
Die Menschenrechte sind allen Personen eigen, unabhängig von Nationalität, Geschlecht, nationaler oder ethnischer Herkunft, Hautfarbe, Religion, Sprache oder sonstigem Status. Sie reichen von den grundlegendsten - dem Recht auf Leben - bis hin zu den Rechten auf Nahrung, Bildung, Arbeit, Gesundheit, gesunde Lebensbedingungen und Freiheit. (Angepasst von Büro des Hohen Kommissars der Vereinten Nationen für Menschenrechte, ohne Datum)

Die Nachhaltigen Entwicklungsziele sind der Plan, um eine bessere und nachhaltigere Zukunft für alle Menschen zu erreichen. Sie adressieren die aktuellen globalen Herausforderungen, einschließlich derer in Bezug auf Armut, Ungleichheit, Klimawandel, Umweltzerstörung, Frieden und Gerechtigkeit. Die 17 Ziele sind alle miteinander verbunden, und um niemanden zurückzulassen, ist es wichtig, dass wir sie alle bis 2030 erreichen. (UN ohne Datum)

Jede professionelle nationale Pflegegruppe, die aufklärt, forscht, bildet und die kontinuierliche Weiterentwicklung von Pflegefachpersonen und der Pflege fördert.

Wertschätzen und Respektieren der Eigenschaften, Merkmale und Präferenzen der Patientin, wie kulturelle und religiöse Überzeugungen, und deren Einbezug in die Planung und Durchführung von Pflege, Dienstleistungen oder der Gestaltung von Programmen.

Informationen, die bei beruflichen Kontakten für eine Person oder Familie privat sind und deren Offenlegung das Recht auf Privatsphäre verletzen, Unannehmlichkeiten, Verlegenheit oder Schaden für die Person oder Familie verursachen können.

Die Pflegefachperson ist eine Person, die eine grundlegende generalistische Pflegeausbildung absolviert hat und von der zuständigen Aufsichtsbehörde in ihrem Land zur Ausübung der Pflege berechtigt ist. Die pflegerische Grundausbildung ist ein formal anerkanntes Ausbildungsprogramm, das eine breite und fundierte, verhaltens-, lebens- und pflegewissenschaftliche Grundlage für die allgemeine Pflegepraxis, für eine Führungsrolle und für die postgraduierte Ausbildung zur spezialisierten oder erweiterten Pflegepraxis bietet. Die Pflegefachperson ist bereit und befugt (1), sich im allgemeinen Bereich der Pflegepraxis zu engagieren, einschließlich Gesundheitsförderung, Krankheitsverhütung und Pflege von körperlich und psychisch kranken sowie behinderten Menschen jeden Alters und in allen Gesundheits- und sonstigen Gemeinschaftssettings; (2) Gesundheitsbildung durchzuführen; (3) sich als Mitglied des Gesundheitsteams uneingeschränkt zu beteiligen;

(4) Pflege- und Gesundheitsassistentinnen zu beaufsichtigen und auszubilden; und (5) an Forschung beteiligt zu sein. (ICN 1987) 
Primäre

Grundversorgung

Privatsphäre

Professionelle

Beziehung

Selbstbestimmung

Selbstreflexion

Soziale Determinanten von Gesundheit

Soziale Gerechtigkeit

Soziale Medien
Die primäre Gesundheitsversorgung ist ein gesamtgesellschaftlicher Ansatz für Gesundheit und Wohlbefinden, der sich an den Bedürfnissen und Vorlieben von Einzelpersonen, Familien und Gemeinschaften orientiert. Sie befasst sich mit den breiteren Determinanten von Gesundheit und konzentriert sich auf die umfassenden und miteinander verbundenen Aspekte der körperlichen, geistigen und sozialen Gesundheit und des Wohlbefindens. (WHO 2019)

Privatsphäre ist das Recht auf Freiheit von Eingriffen in die persönlichen Angelegenheiten, Informationen oder den eigenen Körper.

Eine professionelle Beziehung ist ein ständiger Austausch zwischen zwei Personen, die eine Reihe von festgelegten Einschränkungen oder Grenzen einhält, die nach geltenden ethischen Standards als angemessen erachtet werden.

Das Recht, dass die eigenen Entscheidungen respektiert werden. Selbstbestimmung ist nicht absolut. Sie kann durch kognitive oder affektive Unfähigkeit, Minderjährigkeit, die Gefahr, sich selbst oder andere zu schädigen oder die Freiheit anderer zu beeinträchtigen, eingeschränkt sein.

Die Fähigkeit, das eigene Denken, Planen und Handeln in Bezug auf ethische Verantwortlichkeiten und ethische Leitlinien zu bewerten.

Die Bedingungen, unter denen Menschen geboren werden, aufwachsen, leben, arbeiten und altern. Diese Umstände werden durch die Verteilung von Geld, Macht und Ressourcen auf globaler, nationaler und lokaler Ebene geprägt. Die sozialen Determinanten von Gesundheit sind hauptsächlich für gesundheitliche Ungleichheiten verantwortlich, d. h. ungerechte und vermeidbare Unterschiede im Gesundheitszustand innerhalb und zwischen Ländern. (WHO 2020)

Gleichbehandlung und Gleichberechtigung für Gesellschaft und Beruf (ICN-Strategieplan 2019-2023). Soziale Gerechtigkeit ist eine Form der Fairness, die eine unparteiische Verteilung sozialer Güter und Leistungen sowie eine ebenso unparteiische Verteilung sozialer Lasten erfordert und universelle Menschenrechte bekräftigt. Soziale Ungleichheiten dürfen nur existieren, wenn sie den am wenigsten Begünstigten in der Gesellschaft zugute zu kommen. Soziale Gerechtigkeit gilt für alle Personen, egal ob Staatsbürger oder Nicht-Staatsbürger.

Soziale Medien ist ein Überbegriff, der verwendet wird, um soziale Interaktion durch technologiebasierte Anwendungen zu beschreiben, von denen viele online sind. Dies umfasst Internetforen, Blogs und Netzwerkseiten wie Facebook, Twitter, Instagram und Linkedln, ist aber nicht auf diese begrenzt. (Institute of Business Ethics 2019) 
Umweltgerechtigkeit strebt eine gerechte Verteilung der Vorteile (z. B. sauberes Wasser, Grünflächen, saubere Luft) und eine sichere und gerechte Verteilung der Lasten (z. B. Giftmüllentsorgung, Lärm, Luftverschmutzung durch Fabriken) an. Das beinhaltet Nachhaltigkeit, repräsentative Beteiligung und die Vermeidung von Diskriminierung bei der Verteilung von Umweltbelastungen.

Vertraulichkeit

Vertraulichkeit bezieht sich auf die Pflicht der Pflegefachpersonen, Patientinnendaten nicht an Dritte weiterzugeben, die nicht an der Versorgung der Patientinnen beteiligt sind. Vertraulichkeit ist eine begrenzte Pflicht, manchmal kann sie durch Gesetze oder Vorschriften außer Kraft gesetzt werden, z.B. die vorgeschriebene Meldung von bestimmten Krankheiten.

Verwandte Gruppen

Andere Pflegefachpersonen, Gesundheits- oder weitere Fachpersonen, die einer Person, Familie oder Gemeinschaft Versorgung bieten und auf die gewünschten Ziele hinarbeiten.

Verweigerung aus Gewissensgründen

Werte
Verweigerung der Teilnahme an erforderlichen Maßnahmen oder Antrag auf Befreiung von der Teilnahme an Interventionstypen (z. B. Abtreibung, geschlechtsangleichende Operation, Organtransplantation), die das Gefühl der moralischen Integrität einer Person gefährden. Dazu gehört auch die Weigerung, sich an einer Handlung oder Intervention zu beteiligen, die für eine bestimmte Patientin als unangemessen empfunden wird oder den Willen der Patientin ignoriert.

Werte in der Pflege sind jene Ziele, die sowohl vom Beruf als auch in der Pflegefachperson-Patientin-Beziehung angestrebt werden. Dazu gehören zum Beispiel Gesundheit, Würde, Respekt, Mitgefühl, Gleichbehandlung, Inklusion. Beachte, dass einige Werte (Ziele) auch Charaktereigenschaften (Tugenden) sind. 


\section{REFERENZEN}

Institute of Business Ethics (2019). The Ethical Challenges and Opportunities of Social Media Use. Business Ethics Briefing. 2 May 2019. Retrieved from: https://www.ibe.org.uk/ resource/the-ethical-challenges-and-opportunities-of-social-media-use.html

International Council of Nurses (1987). Definition of a nurse. Available at: https://www.icn.ch/nursing-policy/nursing-definitions

Office of the High Commissioner for Human Rights (n.d.). What are human rights? Retrieved from: https://www.ohchr.org/en/issues/pages/whatarehumanrights.aspx

Schweizerische Eidgenossenschaft (o.D.). 17 Ziele für nachhaltige Entwicklung. Retrieved from: https://www.eda.admin.ch/agenda2030/de/home/agenda-2030/die17-ziele-fuer-eine-nachhaltige-entwicklung.html.

United Kingdom Nursing \& Midwifery Council (2021). What is fitness to practice? Retrieved from: https://www.nmc.org.uk/concerns-nurses-midwives/dealing-concerns/ what-is-fitness-to-practisel

United Nations (n.d.). About the Sustainable Development Goals. Retrieved from: https://www.un.org/sustainabledevelopment/sustainable-development-goals/

Woodbury MG \& Kuhnke JL (2014). Evidence-based practice vs Evidence-informed practice. What's the Difference? Wound Care Canada. Vol 12, Number q, Spring 2014. Retrieved from: https://torontocentreforneonatalhealth.com/wp-content/uploads/2019/09/ Article-Whatsthedifference.pdf

World Health Organization (2019). Primary Health Care Key Facts. Retrieved from https://www.who.int/news-room/fact-sheets/detail/primary-health-care

World Health Organization (2020). Social determinants of health. Retrieved from: https://www.who.int/news-room/q-a-detail/social-determinants-of-health-key-concepts. 




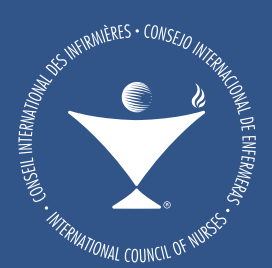

\section{DBfK • ÖGKV • SBK}

\section{c/o DBfK}

Alt-Moabit 91

10555 Berlin, Deutschland

+49302191570

dbfk@dbfk.de

www.dbfk.de $\bullet$ www. öegkv.at $\bullet$ www. sbk.ch 